\title{
Study of transient heat conduction in 2.5D domains using the boundary element method
}

\author{
Luís Godinho*, António Tadeu, Nuno Simões \\ Department of Civil Engineering, University of Coimbra, Polo II-Pinhal de Marrocos, Coimbra 3030-290, Portugal
}

Received 14 April 2003; revised 29 September 2003; accepted 30 September 2003

\begin{abstract}
This paper presents the solution for transient heat conduction around a cylindrical irregular inclusion of infinite length, inserted in a homogeneous elastic medium and subjected to heat point sources placed at some point in the host medium. The solution is computed in the frequency domain for a wide range of frequencies and axial wavenumbers, and time series are then obtained by means of (fast) inverse Fourier transforms into space-time.

The method and the expressions presented are implemented and validated by applying them to a cylindrical circular inclusion placed in an infinite homogeneous medium and subjected to a point heat source, for which the solution is calculated in closed form.

The boundary elements method is then used to evaluate the temperature field generated by a point source in the presence of a cylindrical inclusion, with a non-circular cross-section, inserted in an unbounded homogeneous medium. Simulation analyses using this model are then performed to study the transient heat conduction in the vicinity of these inclusions.
\end{abstract}

(C) 2003 Elsevier Ltd. All rights reserved.

Keywords: Transient heat conduction; Cylinder; Fourier transform; 2.5D problem

\section{Introduction}

Carslaw and Jaeger's book [1] is a reference work on heat transfer, containing analytical solutions and Green's functions for the diffusion equation. In the same work, an extensive survey of numerical methods applicable in the study of this phenomenon is also presented. These are usually grouped by the manner they deal with the timedependent terms. One of these is a 'time marching' approach, with the solution being evaluated step by step, at successive time intervals, starting from a specified initial state of the system. Another approach makes use of the Laplace transform of the time domain diffusion equation, which becomes elliptical. A numerical transform inversion can be used to calculate the physical variables in the time domain, after the solution being obtained for a sequence of values of the transform parameter.

A variety of numerical techniques have been proposed to model and analyze the heat transfer, such as the finite elements [2], the finite differences [3] and the boundary

\footnotetext{
* Corresponding author.

E-mail address: 1godinho@dec.uc.pt (L. Godinho).
}

elements method [4]. Among these techniques, the Boundary Element Method (BEM) is possibly the method best suited to analyze infinite or semi-infinite domains, since it automatically satisfies the far field conditions and only requires a discretization of the interior boundaries of the problem, while the finite elements and the finite differences methods require the full discretization of the domain being studied, which entails highly expensive numerical computational schemes.

The BEM allows a compact description of the region, discretizing only the material discontinuities. Although, the BEM leads to a fully populated system of equations, as opposed to the sparse system given by the finite difference and finite element schemes. The technique is efficient because it substantially reduces the size of the system of equations that needs to be solved. It is well known that the BEM uses the appropriate fundamental solutions, or Green's functions, to relate the field variables in a homogeneous medium to point sources placed within it. The fundamental solution most often used is that for an infinite homogeneous space, because it is known in closed form and has a relatively simple structure. One of the drawbacks of the BEM is that it can only be applied to 
more general geometry and media when the fundamental solution is known, to avoid the discretization of the boundary interfaces, and this may not be possible.

In the 'time marching' approach, the BEM is used to obtain the solution at each time step directly in the time domain. The first time domain direct boundary integral method was proposed by Chang et al. [5] to study planar transient heat conduction. Shaw [6] employed also a timedependent fundamental solution for studying three-dimensional (3D) bodies. Later, Wrobel and Brebbia [7] implemented the BEM for axisymmetric diffusion problems. Dargush and Banerjee [8] also presented a BEM approach in the time domain, where planar, 3D and axisymmetric analyses are all addressed with a time domain convolution.

The dual reciprocity method is another approach, which takes the time derivative in the diffusion equation as a body force and employs the time-independent fundamental solution to Laplace's equation to generate a boundary integral equation [9]. This boundary integral equation can then be solved using a time and space discretization. Different time-marching schemes can be used, based on the way the values of temperature and flux up to actual instant time are computed when solving for a new time step [10-13].

A drawback of the 'time marching' schemes is that they can lead to unstable solutions. An option is to transform the time in a transform variable. The first boundary integral representation for the transient heat conduction analysis, based on the Laplace transform, has been proposed by Rizzo and Shippy [14]. Their numerical approach used a Laplace transform to produce time-independent boundary integration in the transform domain. Since then, different authors presented different solutions for the diffusion type problem using Laplace transforms such as those presented by Cheng et al. [15] and Zhu et al. [16,17]. Recently, Sutradhar et al. [18] used a Laplace transform BEM approach to solve the $3 \mathrm{D}$ transient heat conduction in functionally graded materials, with thermal conductivity and heat capacitance varying exponentially in one coordinate.

A major drawback of using Laplace transforms is the accuracy loss in the inversion process, which magnifies small truncation errors. Different researchers have addressed this problem over the years, and a stable algorithm has been proposed by Stehfest's [19].

Most of the previous approaches have used the Laplace transform to move the solution from the time domain to a transform domain. In the present work, the time Fourier Transform is used to compute the transient heat conduction around cylindrical irregular inclusions of infinite length, located inside a homogeneous elastic medium and heated by point sources placed at the host medium. A spatial Fourier transform in the direction in which the geometry does not vary (the $z$-direction) is used to calculate the response, requiring the solution of a sequence of two-dimensional
(2D) problems with different spatial wavenumbers $k_{z}$. Finally, inverse space Fourier transforms are used to compute the full 3D field.

This solution is known in closed form for inclusions with simple geometry, such as a circular cylinder, for which the wave equation is separable. However, if the inclusion has an irregular cross-section the solution is more difficult to obtain. This paper presents the solution obtained for different cylindrical inclusions, buried in a homogeneous solid medium and subjected to heat point sources placed at some point in the solid, using boundary elements. The inclusions can be of three different types: a solid inclusion, a cavity with prescribed null heat fluxes across its boundary and a cavity with prescribed null temperature along its boundary.

The solution at each frequency is expressed in terms of waves with varying wavenumber $k_{z}$, which is subsequently Fourier transformed into the spatial domain. The wavenumber transform in discrete form is obtained by considering an infinite number of virtual heat point sources equally spaced along the $z$-axis and at a sufficient distance from each other to avoid spatial contamination [20]. Time series are then obtained by means of (fast) inverse Fourier transforms into space-time, using complex frequencies to avoid the aliasing phenomena. In addition, the use of complex frequencies shifts down the frequency axis, in the complex plane, in order to remove the singularities on (or near) the axis and to minimize the influence of the neighboring fictitious sources.

The method presented is implemented and validated by applying it to a cylindrical circular inclusions submerged in an infinite homogenous fluid medium subjected to a point heat source for which the solution is calculated in closed form.

The remainder of this paper is organized as follows: the basic equations of the diffusion problem are first described; then, the paper indicates the main integrals required to solve the BEM, including the necessary Green's functions; a brief validation of the BEM formulation is presented, using circular cylindrical inclusions, subjected to steady-state heat diffusion, for which analytical solutions are known; the proposed BEM model is then used to simulate the heat propagation in the vicinity of different buried inclusions. Frequency and time responses are computed over a grid of receivers for different spatially sinusoidal harmonic line heat sources.

\section{3D problem formulation}

The transient heat conduction in a homogeneous, isotropic body is described by the diffusion equation

$$
\nabla^{2} T=\frac{1}{K} \frac{\partial T}{\partial t}
$$


where

$\nabla^{2}=\left(\frac{\partial^{2}}{\partial x^{2}}+\frac{\partial^{2}}{\partial y^{2}}+\frac{\partial^{2}}{\partial z^{2}}\right)$

$t$ is time, $T(t, x, y, z)$ is temperature, $K=k / \rho c$ is the thermal diffusivity, $k$ is the thermal conductivity, $\rho$ is the density and $c$ is the specific heat. Making use of a Fourier transform in the time domain this equation can be written in the frequency domain as

$\left(\nabla^{2}+\left(\sqrt{\frac{-\mathrm{i} \omega}{K}}\right)^{2}\right) \hat{T}(\omega, x, y, z)=0$

where $i=\sqrt{-1}$ and $\omega$ is the frequency.

Assuming that the geometry of the problem remains constant along one direction $(z)$, the full 3D solution can be attained as a summation of simpler 2D solutions. This procedure requires the application of a Fourier transform along that direction (Tadeu and Kausel [21]). Each 2D solution is computed for a different spatial wavenumber $k_{z}$

$\left(\tilde{\nabla}^{2}+\left(\sqrt{\frac{-\mathrm{i} \omega}{K}-\left(k_{z}\right)^{2}}\right)^{2}\right) \tilde{T}\left(\omega, x, y, k_{z}\right)=0$

with

$\tilde{\nabla}^{2}=\left(\frac{\partial^{2}}{\partial x^{2}}+\frac{\partial^{2}}{\partial y^{2}}\right)$

Applying an inverse Fourier transform along $k_{z}$, the full 3D heat field is obtained. Assuming the existence of virtual sources equally spaced, $L$, along $z$, this inverse Fourier transformation becomes discrete, which allows the solution to be computed by solving a limited number of $2 \mathrm{D}$ problems

$\hat{T}(\omega, x, y, z)=\frac{2 \pi}{L} \sum_{m=-M}^{M} \tilde{T}\left(\omega, x, y, k_{z m}\right) \mathrm{e}^{-i k_{z m} z}$

with $k_{z m}$ being the axial wavenumber given by $k_{z m}=(2$ $\pi / L) m$. The distance $L$ must be sufficiently large to avoid spatial contamination from the virtual sources. The authors have used a similar technique in the analysis of wave propagation inside seismic prospecting boreholes [22] and outdoor propagation of sound waves in the presence of obstacles [23].

\section{Boundary element formulation}

This section will describe the BEM formulation used to obtain the 3D heat field generated by a heat point source placed in the vicinity of a cylindrical inclusion with an irregular shape. Three different types of inclusions will be modeled, namely a solid inclusion, a cavity with null fluxes and a cavity with null temperatures prescribed along its boundary.

As explained before, the problem can be solved as a discrete summation of 2D BEM solutions for different $k_{z}$ wavenumbers, because the geometry of the problem does not change along one direction (the $z$-direction). Then, using the inverse Fourier transform, the $3 \mathrm{D}$ field can be synthesized. The wavenumber transform is obtained in discrete form, as explained above, by considering an infinite number of virtual point sources spaced at equal intervals along the $z$-axis and at a sufficient distance from each other to avoid spatial contamination [24].

Since the literature on the BEM is comprehensive, we do not give full details of the formulation required for the type of problem presented here [9]. Only a brief description of the BEM formulation required to solve each $2 \mathrm{D}$ problem is given.

\subsection{Solid inclusion}

For frequency domain analysis, the temperature $(\tilde{T})$ at any point of the spatial domain can be calculated making use of the Helmoltz equation

$\nabla^{2} \tilde{T}\left(x, y, \omega, k_{z}\right)+\left(\sqrt{\frac{-\mathrm{i} \omega}{K}-\left(k_{z}\right)^{2}}\right)^{2} \tilde{T}\left(x, y, \omega, k_{z}\right)=0$

where

$\nabla^{2}=\left(\frac{\partial^{2}}{\partial x^{2}}+\frac{\partial^{2}}{\partial y^{2}}\right)$

Considering a homogeneous isotropic solid medium of infinite extent, containing an inclusion of volume $V$, bounded by a surface $S$, and subjected to an incident heat wavefield given by $\tilde{T}^{\text {inc }}$, the boundary integral equations can be constructed by applying the reciprocity theorem, leading to

along the exterior domain

$$
\begin{aligned}
& c \tilde{T}^{(\mathrm{ext})}\left(x_{0}, y_{0}, k_{z}, \omega\right) \\
& =\int_{S} q^{(\mathrm{ext})}\left(x, y, \nu_{n}, k_{z}, \omega\right) G^{(\mathrm{ext})}\left(x, y, x_{0}, y_{0}, k_{z}, \omega\right) \mathrm{d} s \\
& \quad-\int_{S} H^{(\mathrm{ext})}\left(x, y, \nu_{n}, x_{0}, y_{0}, k_{z}, \omega\right) \tilde{T}^{(\mathrm{ext})}\left(x, y, k_{z}, \omega\right) \mathrm{d} s \\
& \quad+\tilde{T}_{\mathrm{inc}}\left(x_{0}, y_{0}, k_{z}, \omega\right)
\end{aligned}
$$

along the interior domain

$$
\begin{aligned}
& c \widetilde{T}^{\text {(int) }}\left(x_{0}, y_{0}, k_{z}, \omega\right) \\
& =\int_{S} q^{(\mathrm{int})}\left(x, y, \nu_{n}, k_{z}, \omega\right) G^{(\mathrm{int})}\left(x, y, x_{0}, y_{0}, k_{z}, \omega\right) \mathrm{d} s \\
& \quad-\int_{S} H^{(\mathrm{int})}\left(x, y, \nu_{n}, x_{0}, y_{0}, k_{z}, \omega\right) \tilde{T}^{(\mathrm{int})}\left(x, y, k_{z}, \omega\right) \mathrm{d} s
\end{aligned}
$$

In these equations, superscripts int and ext correspond to the interior and exterior domain, respectively, $\nu_{n}$ is the unit outward normal along the boundary, $G$ and $H$ are, respectively, the fundamental solutions (Green's functions) for the temperature $(\tilde{T})$ and heat flux $(q)$, at $(x, y)$ due to a virtual point heat load at $\left(x_{0}, y_{0}\right)$. The factor $c$ is a constant defined by the shape of the boundary, receiving the value 
$1 / 2$ if $\left(x_{0}, y_{0}\right) \in S$ and is smooth. If the boundary is discretized into $N$ straight boundary elements, with one nodal point in the middle of each element, Eqs. (6) and (7) take the form,

along the exterior domain

$\sum_{l=1}^{N} q^{(\mathrm{ext}) l} G^{(\mathrm{ext}) k l}-\sum_{l=1}^{N} \tilde{T}^{(\mathrm{ext}) l} H^{(\mathrm{ext}) k l}+\tilde{T}_{\mathrm{inc}}^{k}=c_{k} \tilde{T}^{(\mathrm{ext}) k}$

along the interior domain

$\sum_{l=1}^{N} q^{(\mathrm{int}) l} G^{(\mathrm{int}) k l}-\sum_{l=1}^{N} \tilde{T}^{(\mathrm{int}) l} H^{(\mathrm{int}) k l}=c_{k} \tilde{T}^{(\mathrm{int}) k}$

with $q^{(\mathrm{ext}) k}$ and $\tilde{T}^{(\mathrm{ext}) k}$ being the nodal heat fluxes and temperatures at element $k$ in the exterior domain, and $q^{\text {(int)k }}$ and $\tilde{T}^{\text {(int)k }}$ being the nodal heat fluxes and temperatures at element $k$ in the interior domain

$H^{(\mathrm{ext}) k l}=\int_{C_{l}} H^{(\mathrm{ext})}\left(x_{l}, y_{l}, \nu_{l}, x_{k}, y_{k}, k_{z}, \omega\right) \mathrm{d} C_{l}$

$H^{(\mathrm{int}) k l}=\int_{C_{l}} H^{(\mathrm{int})}\left(x_{l}, y_{l}, \nu_{l}, x_{k}, y_{k}, k_{z}, \omega\right) \mathrm{d} C_{l}$

$G^{(\mathrm{ext}) k l}=\int_{C_{l}} G^{(\mathrm{ext})}\left(x_{l}, y_{l}, x_{k}, y_{k}, k_{z}, \omega\right) \mathrm{d} C_{l}$

$G^{(\mathrm{int}) k l}=\int_{C_{l}} G^{(\mathrm{int})}\left(x_{l}, y_{l}, x_{k}, y_{k}, k_{z}, \omega\right) \mathrm{d} C_{l}$

where $\nu_{l}$ is the unit outward normal for the $l$ th boundary segment $C_{l}$. In Eqs. (8) and (9), $H^{(\mathrm{ext})}\left(x_{l}, y_{l}, \nu_{l}, x_{k}, y_{k}, k_{z}, \omega\right)$ and $G^{(\mathrm{ext})}\left(x_{l}, y_{l}, x_{k}, y_{k}, k_{z}, \omega\right)$ are, respectively, the Green's functions for heat fluxes and temperatures components in the exterior medium of the inclusion, while $H^{\text {(int) }}\left(x_{l}, y_{l}, \nu_{l}, x_{k}, y_{k}, k_{z}, \omega\right)$ and $G^{\text {(int) }}\left(x_{l}, y_{l}, x_{k}, y_{k}, k_{z}, \omega\right)$ are, respectively, the Green's functions for heat fluxes and temperatures components in the interior medium of the inclusion, at point $\left(x_{l}, y_{l}\right)$, caused by a concentrated heat load acting at the source point $\left(x_{k}, y_{k}\right)$. The factor $c_{k}$ takes the value $1 / 2$ when the loaded element coincides with the element being integrated.

The required two-and-a-half dimensional Green's functions, for temperature and heat flux in Cartesian coordinates, are those for an unbounded solid medium,

$$
G\left(x_{l}, y_{l}, x_{k}, y_{k}, k_{z}, \omega\right)=\frac{-\mathrm{i}}{4 k} H_{0}\left(\sqrt{\frac{-\mathrm{i} \omega}{K}-\left(k_{z}\right)^{2} r}\right)
$$

$H\left(x_{l}, y_{l}, \nu_{l}, x_{k}, y_{k}, k_{z}, \omega\right)$

$$
=\frac{\mathrm{i}}{4 k} \sqrt{\frac{-\mathrm{i} \omega}{K}-\left(k_{z}\right)^{2}} H_{1}\left(\sqrt{\frac{-\mathrm{i} \omega}{K}-\left(k_{z}\right)^{2} r}\right) \frac{\partial r}{\partial \nu_{l}}
$$

in which $r=\sqrt{\left(x_{l}-x_{k}\right)^{2}+\left(y_{l}-y_{k}\right)^{2}}$ and where $H_{n}()$ are Hankel functions of the second kind and order $n$. The thermal diffusivity and the thermal conductivity in these equations are the ones associated with the exterior and the interior material of the inclusion when incorporated in Eqs. (8) and (9), respectively.

The integrations in Eqs. (8) and (9) are evaluated using a Gaussian quadrature scheme, when they are not performed along the loaded element. For the loaded element, the existing singular integrands in the source terms of the Green's functions are calculated in closed form [25,26].

The final integral equations are manipulated and combined so as to impose the continuity of temperatures and heat fluxes along the boundary of the inclusion, to establish a system of equations. The solution of this system of equations gives the nodal temperatures and heat fluxes, which allow the reflected heat field to be defined.

\subsection{Cavity with null fluxes along its boundary}

In this case, the boundary conditions prescribe null normal heat fluxes along the boundary $S$. Thus, Eq. (6) is simplified to

$$
\begin{aligned}
& c \tilde{T}^{(\mathrm{ext})}\left(x_{0}, y_{0}, k_{z}, \omega\right) \\
& =-\int_{S} H^{(\mathrm{ext})}\left(x, y, \nu_{n}, x_{0}, y_{0}, k_{z}, \omega\right) \tilde{T}^{(\mathrm{ext})}\left(x, y, k_{z}, \omega\right) \mathrm{d} s \\
& \quad+\tilde{T}_{\mathrm{inc}}\left(x_{0}, y_{0}, k_{z}, \omega\right)
\end{aligned}
$$

The solution of this integral for an arbitrary boundary surface $(S)$ will require again the discretization of the boundary into $N$ straight boundary elements, following a procedure similar to one described above.

\subsection{Cavity with null temperatures along its boundary}

Null temperatures are now prescribed at the surface of the cavity, which leads to the equation

$$
\begin{aligned}
& \int_{S} q^{(\mathrm{ext})}\left(x, y, \nu_{n}, k_{z}, \omega\right) G^{(\mathrm{ext})}\left(x, y, x_{0}, y_{0}, k_{z}, \omega\right) \mathrm{d} s \\
& \quad+\tilde{T}_{\mathrm{inc}}\left(x_{0}, y_{0}, k_{z}, \omega\right)=0
\end{aligned}
$$

The solution of this equation is again obtained as described before.

\section{Responses in the time domain}

The heat in the spatial-temporal domain is obtained by a numerical inverse fast Fourier transform in $k_{z}$ and frequency domain. Complex frequencies with a small imaginary part of the form $\omega_{\mathrm{c}}=\omega-\mathrm{i} \eta$ (with $\eta=0.7 \Delta \omega, \Delta \omega$ being the frequency step) are used to avoid the aliasing phenomena. In the time domain, this shift is later taken into account by applying an exponential window of the form $\mathrm{e}^{\eta t}$ to the response.

The temporal variation of the source can be arbitrary. The application of a time Fourier transformation defines the frequency domain where the BEM solution is required. So, 
the frequency domain may range from $0.0 \mathrm{~Hz}$ to very high frequencies. However, it happens that we may cut-off the upper frequencies of this domain because the heat responses decrease very fast as the frequency increases. The frequency $0.0 \mathrm{~Hz}$ is the static response that can be obtained by limiting the frequency to zero. As we are using complex frequencies, the response can be computed because the argument of the Hankel in Eqs. (8) and (9) is -i $\eta$, that is different than zero.

As stated before, the Fourier transformations are achieved by discrete summations over wavenumbers and frequencies, which is mathematically equivalent to adding periodic sources at spatial intervals $L=2 \pi / \Delta k_{z}$ (in the $z$-axis) and temporal intervals $T=2 \pi / \Delta \omega$, with $\Delta k_{z}$ being the wavenumber step. The spatial separation $L$ must be sufficiently large to avoid contamination of the response by the periodic sources. In other words, the contribution to the response by the fictitious sources must be guaranteed to occur at times later than $T$. This goal can also be aided substantially by shifting the frequency axis slightly downward, that is, by using complex frequencies with a small imaginary $\operatorname{part}\left(\omega_{\mathrm{c}}=\right.$ $\omega-\mathrm{i} \eta)$. This technique results in a significant attenuation or virtual elimination of the periodic sources.

\section{BEM validation}

The BEM algorithm was implemented and validated by applying it to a cylindrical circular inclusion, as in Fig. 1, subjected to a harmonic point heat source applied at point $\mathrm{O}$ $\left(x_{0}, y_{0}\right)$, for which the solution is known in closed form and described in Appendix A. The incident heat field is given by the expression

$$
\begin{aligned}
& \tilde{T}_{\mathrm{inc}}\left(x, y, k_{z}, \omega\right) \\
& \quad=\frac{-\mathrm{i} A}{4 k} H_{0}\left(\sqrt{\frac{-\mathrm{i} \omega}{K}-\left(k_{z}\right)^{2}} \sqrt{\left(x-x_{0}\right)^{2}+\left(y-y_{0}\right)^{2}}\right)
\end{aligned}
$$

where $A(\mathrm{~J} / \mathrm{m})$ is the amplitude of the source.

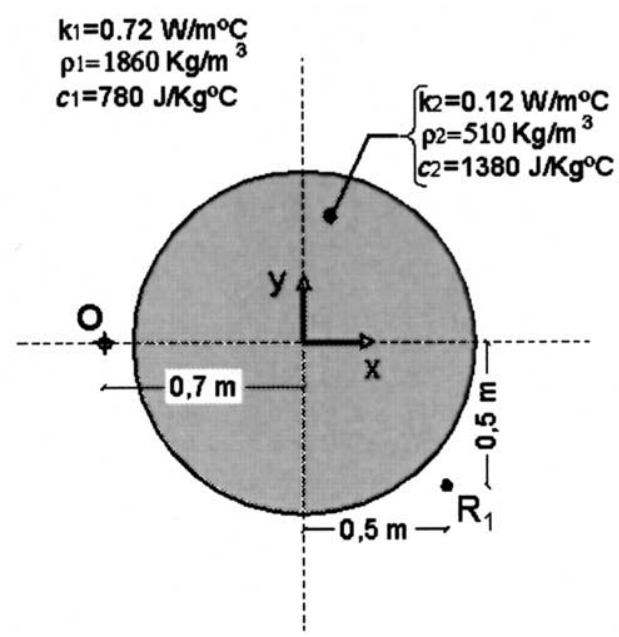

Fig. 1. Cylindrical circular solid inclusion in an unbounded solid medium. Medium properties.
Next, the results are obtained for the three scenarios studied here. First, the inclusion is assumed to be solid and bonded to the exterior domain, allowing the continuity of heat fluxes and temperatures. In a second case, null heat fluxes are imposed at the interface between the cylindrical inclusion and the exterior domain. One last situation refers to a circular cylindrical inclusion with null temperatures along its boundary. For all cases, the thermal properties of the host medium are kept constant, with $k_{1}=0.72 \mathrm{~W} \mathrm{~m}^{-1}{ }^{\circ} \mathrm{C}^{-1}, \quad c_{1}=780 \mathrm{~J} \mathrm{Kg}^{-1}{ }^{\circ} \mathrm{C}^{-1} \quad$ and $\rho_{1}=1860 \mathrm{Kg} \mathrm{m}^{-3}$. When an elastic inclusion is modeled, its properties are assumed to be $k_{2}=0.12 \mathrm{~W} \mathrm{~m}^{-1}{ }^{\circ} \mathrm{C}^{-1}$, $c_{2}=1380 \mathrm{~J} \mathrm{Kg}^{-1}{ }^{\circ} \mathrm{C}^{-1}$ and $\rho_{2}=510 \mathrm{Kg} \mathrm{m}^{-3}$. The simulated systems are heated by a harmonic line source located at $(x=-0.7 \mathrm{~m}, y=0.0 \mathrm{~m})$. All the calculations are performed in the frequency range $\left(0,128 \times 10^{-8} \mathrm{~Hz}\right)$ with a frequency increment of $\Delta \omega=10^{-8} \mathrm{~Hz}$, defining the imaginary part of the frequency to be given by $\eta=0.7$ $\Delta \omega$. The results are computed for two different values of the parameter $k_{z}\left(k_{z}=0.0,1.0 \mathrm{rad} / \mathrm{m}\right)$. Fig. 2 displays the real and imaginary parts of the responses, with the analytical responses being represented by solid lines, and the BEM solutions by marked points. The circle and the triangle marks indicate the real and imaginary parts of the BEM responses, respectively, computed using 100 constant boundary elements.

All the plots reveal an excellent agreement between the two solutions presented. Very good results were also obtained for heat sources and receivers placed at different positions.

\section{Applications}

Next we consider the heat field generated by a cylindrical solid inclusion buried in an unbounded solid medium, with a rectangular cross-section. At time $t=139 \mathrm{~h}$, a point heat source at a point $\mathrm{O}$ creates a spherical heat pulse that evolves as plotted in Fig. 3a, propagating away from $\mathrm{O}$ with a power that increases linearly from 0 to $1000.0 \mathrm{~W}$. The field generated is computed at receivers $R 1, R 2$ and $R 3$, located in three planes equally spaced $(3 \mathrm{~m})$ along the $z$ direction. The geometry of the plane containing the point source is shown in Fig. 3b.

The thermal conductivity $\left(k_{1}=1.4 \mathrm{~W} \mathrm{~m}^{-1}{ }^{\circ} \mathrm{C}^{-1}\right)$, the density $\left(\rho_{1}=2300 \mathrm{Kg} \mathrm{m}^{-3}\right)$ and the specific heat $\left(c_{1}=\right.$ $880.0 \mathrm{~J} \mathrm{Kg}^{-1}{ }^{\circ} \mathrm{C}^{-1}$ ) of the host medium (concrete) are kept constant in all the analyses. The material of the inclusion (steel) has a thermal conductivity $\left(k_{2}\right)$ of $63.9 \mathrm{~W} \mathrm{~m}^{-1} \times$ ${ }^{\circ} \mathrm{C}^{-1}$, a density $\left(\rho_{2}\right)$ of $7832 \mathrm{Kg} \mathrm{m}^{-3}$ and a specific heat $\left(c_{2}\right)$ of $434.0 \mathrm{~J} \mathrm{Kg}^{-1}{ }^{\circ} \mathrm{C}^{-1}$. The computations are performed in the frequency range $\left(0,128 \times 10^{-7} \mathrm{~Hz}\right)$, with a frequency increment of $1 \times 10^{-7} \mathrm{~Hz}$, which determines the total time duration $(T=2778 \mathrm{~h})$ for the analysis in the time domain. The spatial period considered in the analysis 

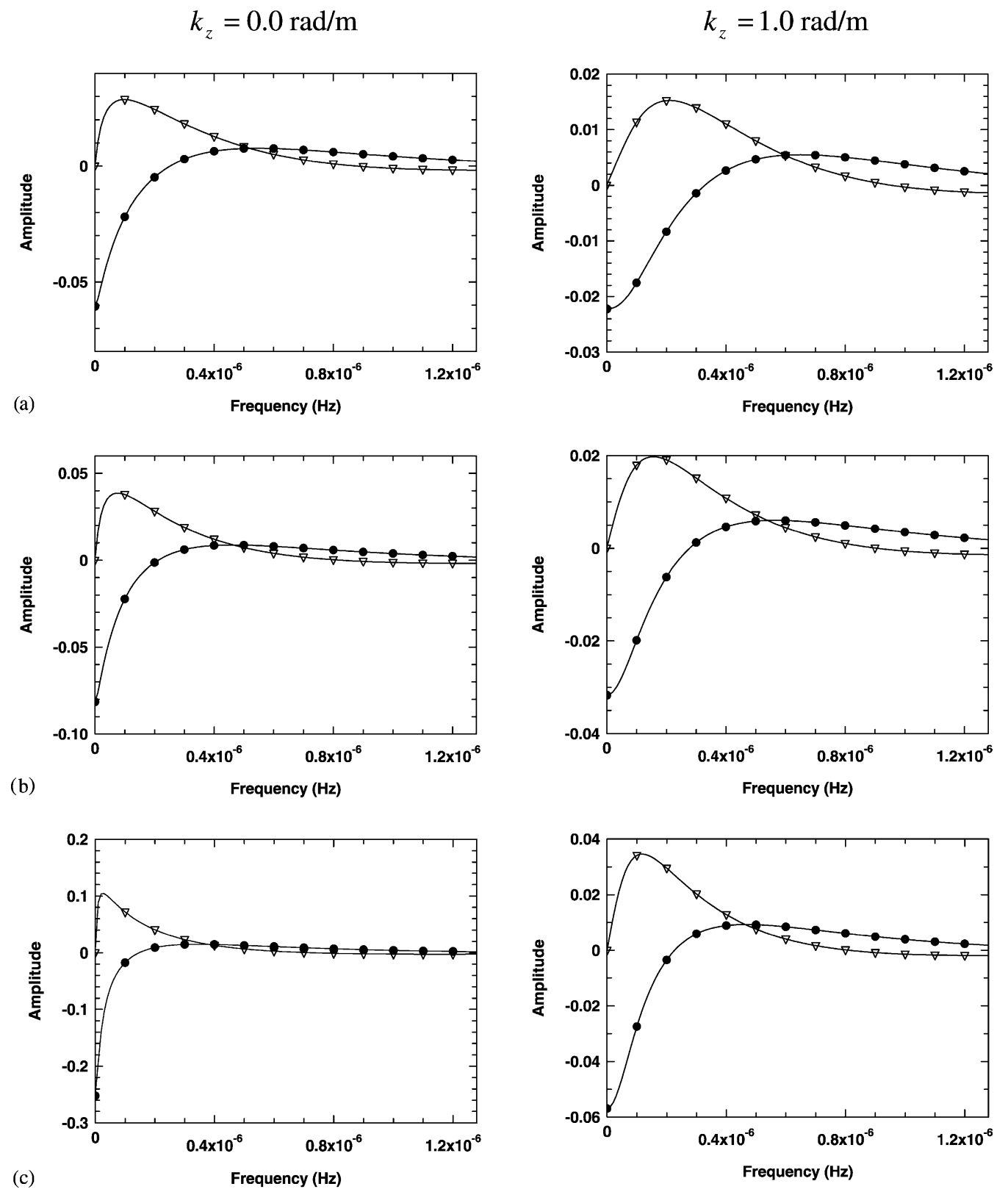

Fig. 2. Real and imaginary parts of the heat responses: (a) Cylindrical circular solid inclusion in an unbounded solid medium; (b) cylindrical circular cavity with null fluxes and (c) cylindrical circular cavity with null temperatures.

is $L=2 \sqrt{k_{2} /\left(\rho_{2} c_{2} \Delta f\right)}=28 \mathrm{~m}$. The inclusion has been modeled with 80 boundary elements.

Figs. 4a shows the results obtained at receivers $R 1, R 2$ and $R 3$. To allow a better understanding of the physics of the problem, the results are compared with those computed at the same receivers for an infinite homogeneous medium, displayed in Fig. 4b. For all plots, the time response begins at null temperature, corresponding to the initial conditions defined for the present problem. As the source starts emitting energy $(t=139.0 \mathrm{~h})$, the temperature at the receivers increases progressively.

The receivers located at $z=0.0 \mathrm{~m}$ (Fig. 4a) are the first to register a clear change in temperature. Of these, receiver
$R 1$, located closest to the heat source, registers the temperature changes most quickly. The temperature registered at this point increases smoothly as the energy generated at the source point increases from 0 to $1000.0 \mathrm{~W}$, reaching approximately $32.0{ }^{\circ} \mathrm{C}$ when the source reaches maximum power $(t \approx 695.0 \mathrm{~h})$. As the source stabilizes at $1000.0 \mathrm{~W}$, the temperature continues to increase at a slower rate, and a maximum value of $39.0{ }^{\circ} \mathrm{C}$ is reached for $t \approx 1250.0 \mathrm{~h}$. At this point, the source power starts to decline until it stops emitting energy completely. The energy introduced at the source point continues to propagate to colder regions in order to establish the equilibrium condition. Since the analysis is performed for 


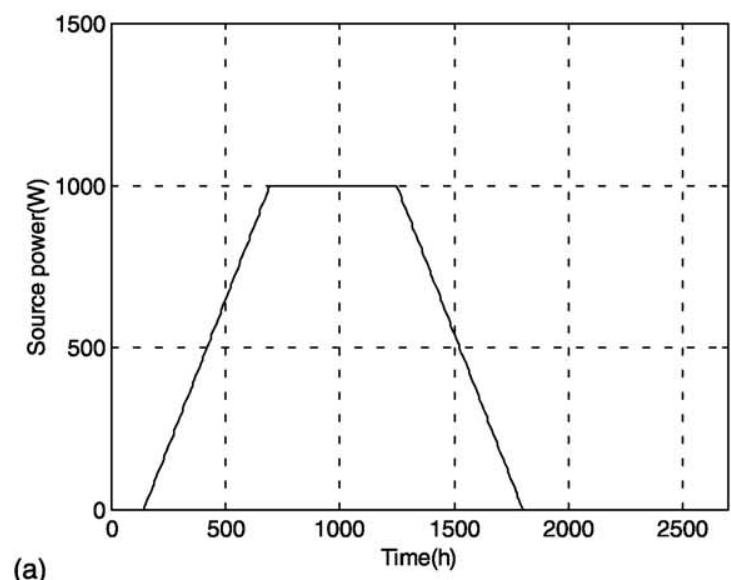

$\mathrm{k} 1=1.4 \mathrm{~W} / \mathrm{m}^{\circ} \mathrm{C}$
$\rho 1=2300 \mathrm{Kg} / \mathrm{m}^{3}$
$\mathrm{c} 1=880 \mathrm{~J} / \mathrm{Kg}^{\circ} \mathrm{C}$

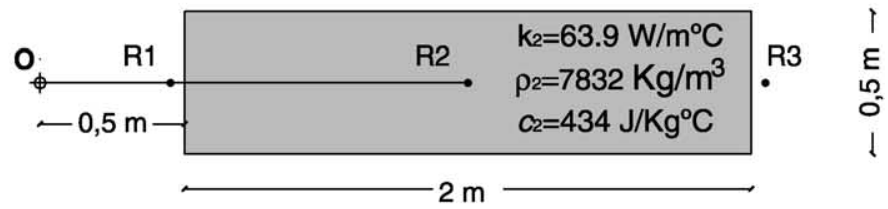

(b)

Fig. 3. (a) Temporal evolution of the heat source and (b) geometry of the problem.

an infinite medium, this equilibrium would be reached only for $t=\infty$. Comparing the response at this receiver with that computed at receiver $R 2$, placed inside the steel inclusion, it is clear that the latter reaches much lower temperatures, not only because it is placed further away from the source but also because it is inside a material with a much higher diffusivity. In fact, the energy reaching the steel is spread over the full section of the block, at higher velocity than in the host medium, allowing a lower but more uniform increase in the registered temperatures. The presence of this steel block also influences the response at receiver $R 3$, for which the temperature rises at almost the same rate as the temperature of the steel block.

The responses computed for the case of an infinite medium further confirm the above explanations. Although, the shape of the temperature curve registered at receiver $R 1$ is similar to the previous case, it reaches a temperature 2.5 times higher than the earlier one. For this case, the energy propagation is determined only by the physical characteristics of the host medium (concrete), and it occurs much more slowly than before. This thermal energy is thus retained for longer at regions near the source, allowing the temperature in this region to reach higher values. The same phenomenon can be observed at receiver $R 2$, which registers temperatures twice as high as those of the first case. The opposite behavior is registered at receiver $R 3$, where the maximum temperatures are lower than those computed in the presence of the steel block. At this receiver, the presence of the steel block allows a larger amount of energy to travel faster to regions behind it. As a consequence, the temperature at this receiver starts increasing earlier, when the steel block is present.

Observing the results computed at receivers placed at $z=3.0 \mathrm{~m}$, when the steel block is present, it can be seen that the evolution of temperature at all three receivers is very similar. In fact, the heat propagation occurs mainly through the most conductive material, which is the steel block. For this reason, the higher temperatures are registered at receiver $R 2$, while the temperatures at the two receivers located in the concrete medium register slightly lower temperatures. Analyzing the behavior of the same receivers in an infinite homogeneous medium, it is evident that the heating curves have a distinct evolution, and the most influential factor is the distance between the receiver and the source. It is also clear that the maximum temperatures occur at later times, since the concrete has a lower diffusivity than the steel. These conclusions are corroborated by receivers placed at $z=6.0 \mathrm{~m}$. At this position, in the presence of the steel inclusion, the heating curves registered at the two receivers located outside the inclusion, $R 1$ and $R 3$, are almost coincident and lower than that observed for the receiver located inside the steel block $(R 2)$.

In Fig. 5, a sequence of snapshots $(t=500,1500$ and $1750 \mathrm{~h}$ ) displays the temperature field along a transversal grid of receivers placed at $z=0.0 \mathrm{~m}$ and a longitudinal grid of receivers placed at $x=-0.75 \mathrm{~m}$. These figures show the resulting temperature fields as contour plots.

As the heat propagates away from the source, the energy spreads out. At time $t=500 \mathrm{~h}$ (Fig. 5a) a large amount of energy generated by the point source has reached the steel block, travelling faster along the longitudinal direction of the inclusion than outside. For the same reason, the regions behind the inclusion, relative to the source, register higher temperatures along the transversal grid of receivers than the other regions, which are at the same distance from the source. As the time progresses, the energy continues to spread through the full domain of receivers, generating a progressive temperature increase. For $t=1500 \mathrm{~h}$ (Fig. 5b), this temperature increase is visible at both grids of receivers. Analyzing the temperature field along the longitudinal grid, it is possible to confirm that the presence of the steel block has allowed a large amount of energy to reach receivers at large distances from the source. In fact, even for points located at $z=6.0 \mathrm{~m}$, it is possible to observe a distinct rise in temperature. As the source power drops to $0 \mathrm{~W}$, the energy continues to propagate through the media, with a consequent temperature increase for receivers located further away from the source, and a fall in temperature at 


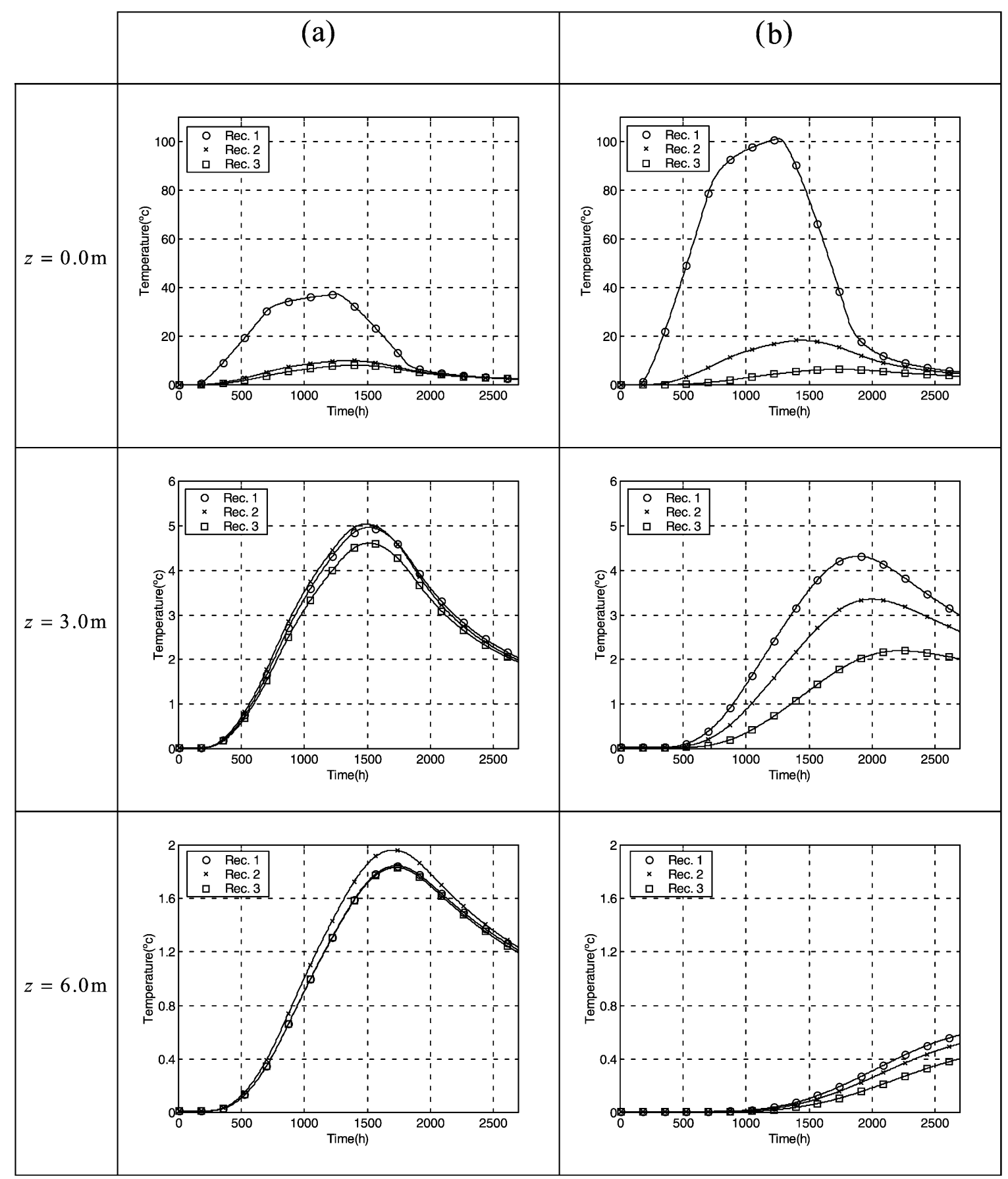

Fig. 4. Heat curves registered at $R 1, R 2$ and $R 3$ for different $z$-coordinates: (a) homogeneous concrete medium with rectangular inclusion and (b) infinite homogeneous concrete medium.

receivers located closer to it. This is visible for $t=1750 \mathrm{~h}$ (Fig. 5c), at receivers placed along the longitudinal grid. Receivers placed further away from the heat source in the $z$ direction are still exhibiting a slight temperature increase, while those placed closer to the source have already suffered a significant fall in temperature.

Simulations have also been performed for the case of inclusions with specified boundary conditions of constant flux or temperature, where the concrete host medium maintains the material properties previously defined. Fig. 6 presents the results computed at the receivers $R 1$ and $R 3$ when boundary conditions of either null heat flux (Fig. 6a) or null boundary temperature are ascribed to the boundary of the inclusion (Fig. 6b). Since the receivers $R 2$ are located inside the inclusion, they are not used here.

When null heat fluxes are considered for the boundary of the inclusion and $z=0.0 \mathrm{~m}$, there is a marked difference between the temperatures registered at $R 1$ and $R 3$, with $R 1$ reaching very high temperatures (approximately $145^{\circ} \mathrm{C}$ ) and $R 2$ registering maximum temperatures 


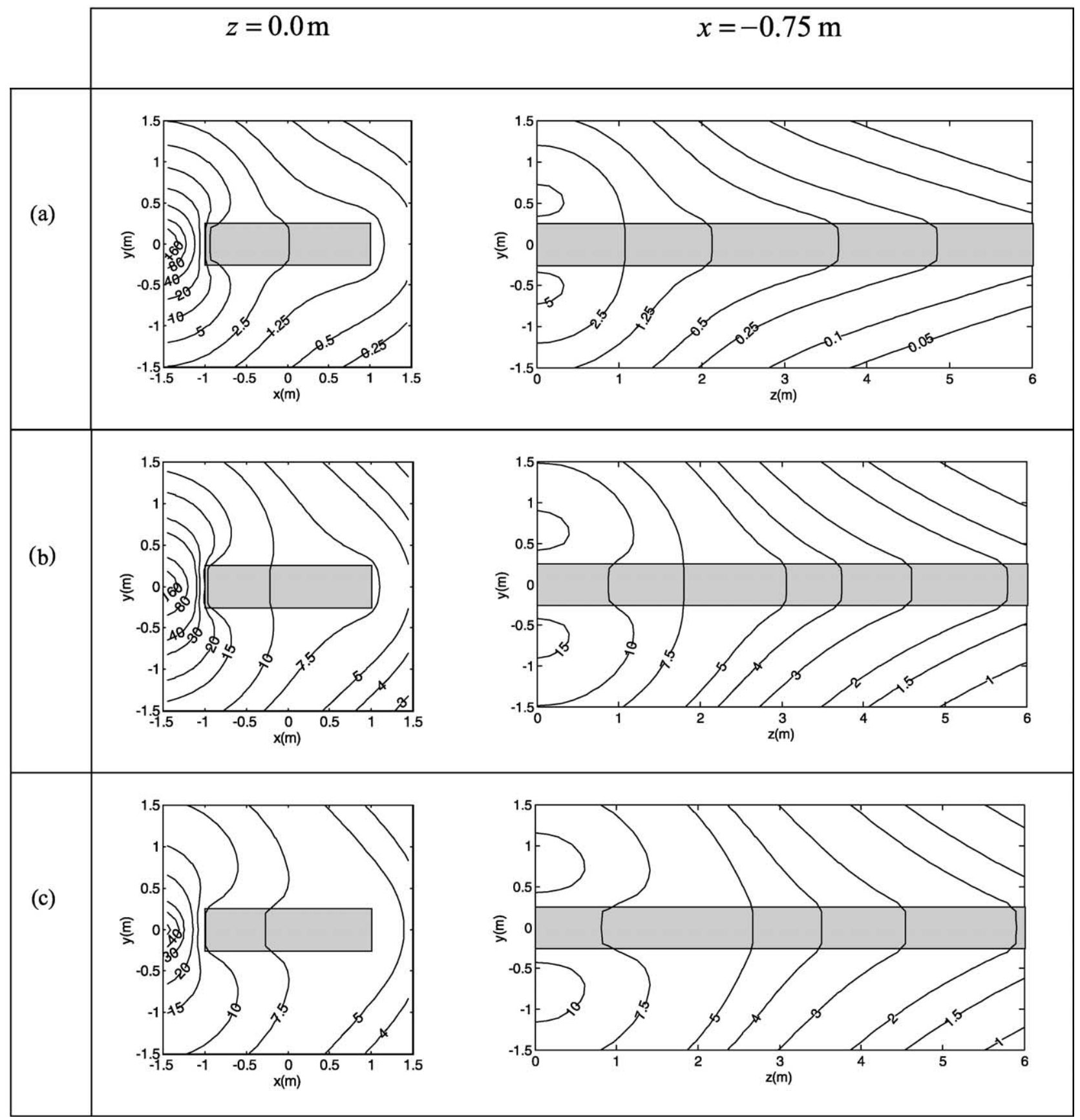

Fig. 5. Temperature fields registered at the two grids of receivers: (a) $t=500 \mathrm{~h}$; (b) $t=1500 \mathrm{~h}$ and (c) $t=1750 \mathrm{~h}$.

close to $5^{\circ} \mathrm{C}$. This behavior can be explained by the fact that, for this case, the inclusion acts as a thermal insulator, since the null flux conditions ascribed to the boundary create a thermal energy concentration on the source side of the inclusion, producing a temperature rise in the region between the source and the inclusion. In contrast, very little energy reaches the region behind the inclusion and the temperature registered there is very low. Comparing these results with those presented in Fig. 4, it is possible to observe that the temperatures registered at receiver $R 1$ are now much higher than in the previous cases, mainly because of thermal energy concentration described above. For receivers placed further away, along the $z$-direction, the temperatures registered at the two receivers tend to approximate between them, although they still diverge relative to those observed in Fig. 4a, for the case of the steel inclusion. Because of the 3D character of the problem, the energy concentration becomes less evident as we advance in $z$, and the solution approaches the one registered for an infinite homogeneous medium, although with temperatures slightly higher at $R 1$ and lower at $R 2$. 


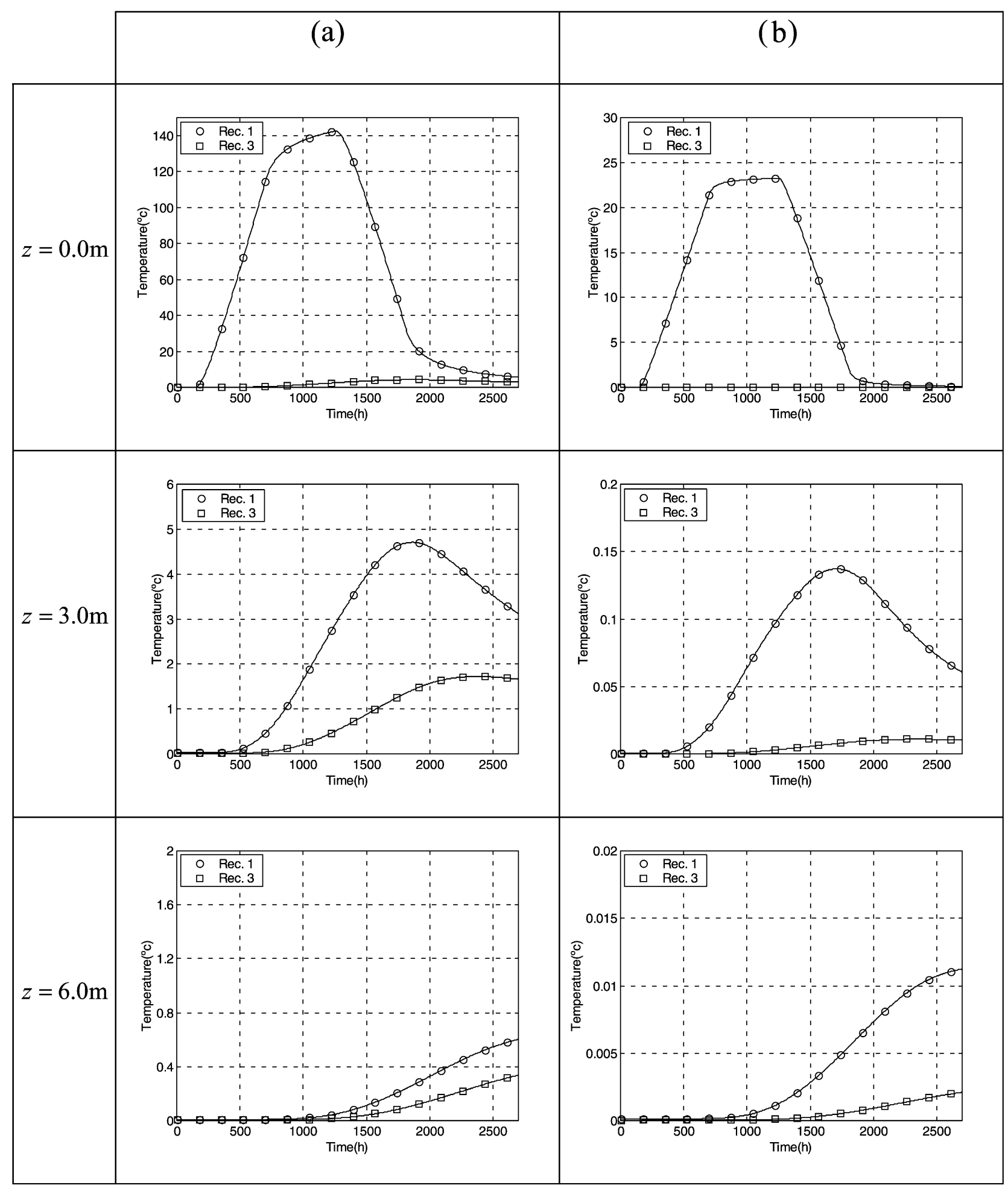

Fig. 6. Heat curves registered at $R 1, R 3$ for different $z$-coordinates: rectangular cavity with (a) null fluxes and (b) null temperatures.

This scenario changes when null temperature conditions are ascribed to the boundary of the inclusion, as shown in Fig. 6b. As expected, receiver $R 1$ still registers higher temperatures than $R 2$, but the maximum temperature registered is now much lower than in the previous case, since it is located close to a null temperature surface. As positions further away in $z$ are considered, the temperature at $R 1$ decreases markedly, reaching maximum values below $0.15^{\circ}$, even when $z=3.0 \mathrm{~m}$. The temperatures registered in $R 2$ are very low, since the receiver is close to a boundary $0^{\circ}$ and it is placed on the opposite side of the inclusion from the source. These low temperatures are even more evident when the receiver is located further away in $z$. 
To better understand the heat propagation phenomenon around inclusions with null flux or null temperature boundary conditions, Fig. 7 present a sequence of snapshots displaying the temperature field along a longitudinal grid of receivers located in the plane $x=-0.75 \mathrm{~m}$. The temperature fields are again displayed as contour plots. At time $t=500 \mathrm{~h}$ (Fig. 7a), the effect of the propagating thermal energy is clearly visible over the grid of receivers, and the results reveal marked differences between the two cases analysed. It is possible to observe that much higher temperatures are registered when null fluxes are prescribed, particularly at receivers placed further away in $z$. In fact, for the second case, the null temperatures prescribed along the surface of the inclusion do not allow the same temperature rise, particularly at larger distances along the $z$-axis and for receivers placed closer to the inclusion. As time advances, this difference remains clearly visible, and for $t=1500 \mathrm{~h}$ (Fig. 7b) the shape of the contour lines in the two plots confirms the different behaviors observed for the two cases. In the first case, the imposition of null fluxes along the boundary allows the energy to be retained in the host medium, while in the second; the boundary with null temperatures generates fluxes that tend to drain energy from the system. This effect is noticeable if one observes the rapid temperature variation in the direction perpendicular to the boundary as receivers placed closer to the inclusion are considered, indicating a strong temperature gradient. When the null fluxes are considered, however, the temperature variation along the same direction is much smaller, and it is much more evident at receivers placed along the direction of the axis of the inclusion. The same behavior can be seen in Fig. 7c for $t=1750 \mathrm{~h}$, after the source stops emitting energy. At this later time, it is interesting to note that, in

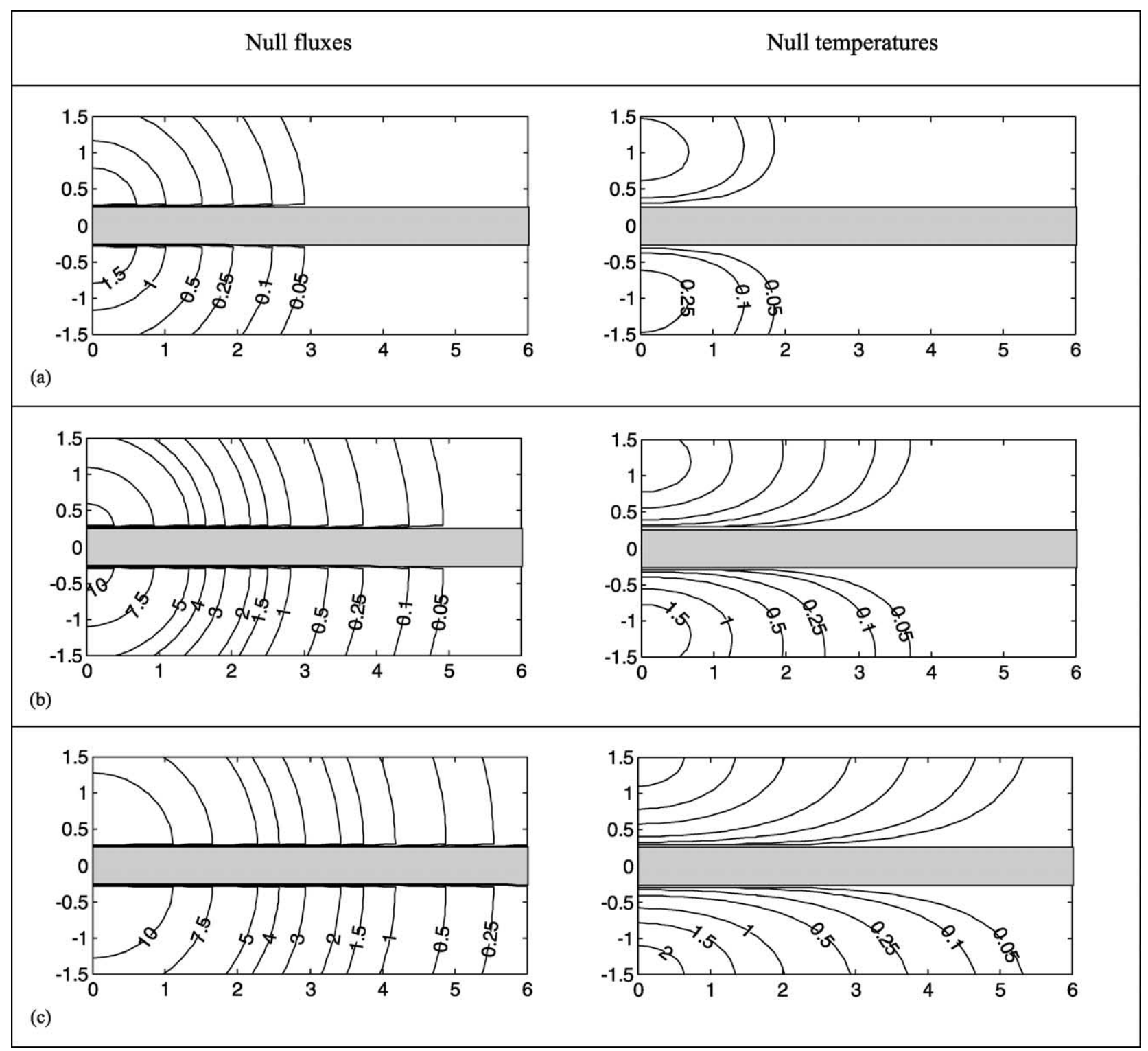

Fig. 7. Temperature fields registered along a longitudinal grid of receivers located in the plane $x=-0.75 \mathrm{~m}$ : (a) $t=500 \mathrm{~h}$; (b) $t=1500 \mathrm{~h}$ and (c) $t=1750 \mathrm{~h}$. 
both cases, the temperature continues to rise, since the energy is still propagating away from the source point. Notice that this behavior differs from that found in the presence of a steel inclusion, which exhibits higher diffusivity.

\section{Conclusions}

A discrete integration over wavenumbers and frequencies has been used to compute the 3D heat field generated by harmonic heat point sources placed in the vicinity of a cylindrical irregular inclusion in an unbounded solid medium. The discretization of the wavenumber-frequency integral transform presented is mathematically equivalent to a periodic sequence of sources, parallel to the axis of the cylinder, that are also periodic in time. We have removed the effects of these periodicities by using complex frequencies.

The method was implemented and used to show the main features of the transient heat conduction across media containing an inclusion. The time responses obtained made it possible to confirm that the method presented was useful in the analysis of 3D heat propagation in the presence of a 2D geometry. The results computed for the three situations analysed (a solid inclusion, an inclusion with null surface temperature and an inclusion with null normal fluxes along its surface) have shown marked differences in their behavior and the temperature field was found to be strongly dependent on the prescribed boundary conditions.

\section{Appendix A. Analytical solution of the 3D transient heat transfer through a cylindrical circular solid inclusion}

\section{A.1. Solid inclusion}

Consider a spatially uniform solid medium of infinite extent, having a cylindrical solid inclusion with radius $a$, as shown in Fig. A1. The exterior solid medium (1) exhibits

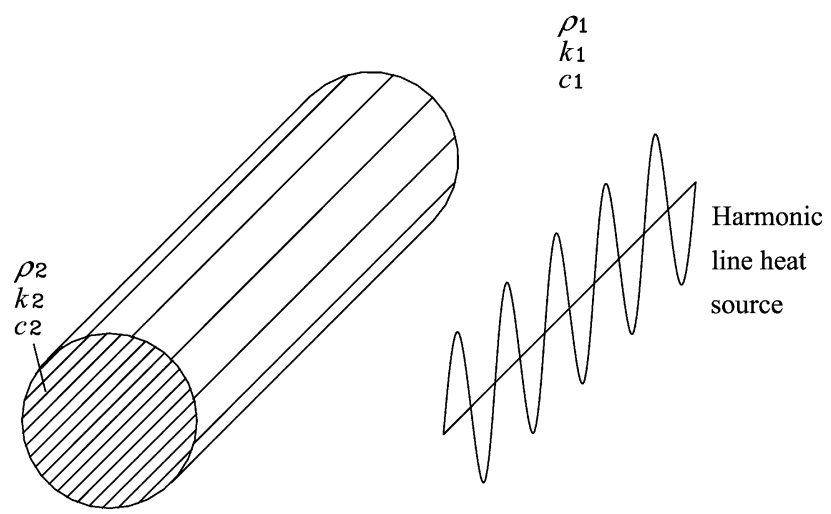

Fig. A1. Geometry of the problem. a thermal conductivity $k_{1}$, a density $\rho_{1}$ and a specific heat $c_{1}$, while the cylindrical inclusion (2) shows a thermal conductivity $k_{2}$, a density $\rho_{2}$ and a specific heat $c_{2}$. This system is subjected to a spatially sinusoidal harmonic heat line source, placed in the exterior medium $\left(x_{0}, 0,0\right)$, oscillating with a frequency $\omega$, of the form $p(x, y, z, t)=$ $\delta\left(x-x_{0}\right) \delta(y) \mathrm{e}^{\mathrm{i}\left(\omega t-k_{z} z\right)}$ with $k_{z}$ being the wavenumber in $z$, and $\delta\left(x-x_{0}\right)$ and $\delta(y)$ being Dirac-delta functions.

\section{A.1.1. Incident heat field (or free-field)}

The 3D incident heat field produced by this heat source can be expressed as

$\tilde{T}_{\text {inc }}\left(\omega, r, k_{z m}\right)=\frac{-\mathrm{i} A}{4 k_{1}} H_{0}\left(k_{\alpha_{1}} r^{\prime \prime}\right)$

where the subscript inc denotes the incident heat field, $A$ is the heat amplitude, $H_{n}(\mathrm{)})$ are Hankel functions of the second kind and order $n$,

$k_{\alpha_{1}}=\sqrt{\frac{-\mathrm{i} \omega}{K_{1}}-\left(k_{z m}\right)^{2}}, \quad K_{1}=\frac{k_{1}}{\rho_{1} c_{1}} \quad$ and

$r^{\prime \prime}=\sqrt{\left(x-x_{0}\right)^{2}+y^{2}}$.

The incident field needs to be expressed in terms of heat waves centered at the axis of the cylinder. Using the Graf's addition theorem [27] one may obtain, leading to the expressions (in cylindrical coordinates)

$$
\begin{aligned}
& \tilde{T}_{\mathrm{inc}}\left(\omega, r, \theta, k_{z m}\right) \\
& \quad=-\frac{\mathrm{i} A}{4 k_{1}} \sum_{n=0}^{\infty}(-1)^{n} \varepsilon_{n} H_{n}\left(k_{\alpha_{1}} r_{0}\right) J_{n}\left(k_{\alpha_{1}} r\right) \cos (n \theta),
\end{aligned}
$$

when $r<r_{0}$

$$
\begin{aligned}
& \tilde{T}_{\mathrm{inc}}\left(\omega, r, \theta, k_{z m}\right) \\
& \quad=-\frac{\mathrm{i} A}{4 k_{1}} \sum_{n=0}^{\infty}(-1)^{n} \varepsilon_{n} J_{n}\left(k_{\alpha_{1}} r_{0}\right) H_{n}\left(k_{\alpha_{1}} r\right) \cos (n \theta),
\end{aligned}
$$

when $r>r_{0}$

in which $r_{0}$ is the distance from the source to the axis of the inclusion, $J_{n}(\ldots)$ are Bessel functions of order $n$ and

$\varepsilon_{n}= \begin{cases}1, & \text { if } n=0 \\ 2, & \text { if } n \neq 0\end{cases}$

\section{A.1.2. Reflected heat field in the exterior region}

The heat generated by this source propagates and hits the surface of the inclusion, where part of the incident heat energy is reflected back, and the remaining energy is transmitted into the solid material inclusion, in the form of propagating heat waves. The reflected heat field in the exterior region can be expressed in a form similar to that of 
the incident field, namely

$\tilde{T}_{\text {ref }}\left(\omega, r, \theta, k_{z m}\right)=\sum_{n=0}^{\infty} A_{n} H_{n}\left(k_{\alpha_{1}} r\right) \cos (n \theta)$

in which the subscript $r e f$ denotes the reflected heat field, $A_{n}$ is as yet unknown coefficient to be determined from appropriate boundary conditions. Together with an implicit factor $e^{\mathrm{i}\left(\omega t-k_{z} z\right)}$, the Hankel functions in Eqs. (A4) represent diverging or outgoing cylindrical heat waves.

\section{A.1.3. Transmitted heat field in the interior region}

The transmitted heat field can be seen as standing heat waves, which can be expressed as

$\tilde{T}_{\text {trans }}\left(\omega, r, \theta, k_{z m}\right)=\sum_{n=0}^{\infty} B_{n} J_{n}\left(k_{\alpha_{2}} r\right) \cos (n \theta)$

in which the subscript trans denotes the reflected heat field

$k_{\alpha_{2}}=\sqrt{\frac{-\mathrm{i} \omega}{K_{2}}-\left(k_{z m}\right)^{2}}, \quad K_{2}=\frac{k_{2}}{\rho_{2} c_{2}}$

$B_{n}$ is again an unknown coefficient to be determined by imposing the appropriate boundary conditions.

\section{A.1.4. Definition of $A_{n}$ and $B_{n}$}

The definition of the appropriate boundary conditions allows $A_{n}$ and $B_{n}$, that is the reflected and transmitted heat field, to be defined. The solution in this case is computed imposing the continuity of temperatures and normal heat fluxes on the solid-solid interface

$$
\tilde{T}_{\text {inc }}\left(\omega, a, \theta, k_{z m}\right)+\tilde{T}_{\text {ref }}\left(\omega, a, \theta, k_{z m}\right)=\tilde{T}_{\text {trans }}\left(\omega, a, \theta, k_{z m}\right)
$$

$$
\begin{gathered}
k_{1} \frac{\partial\left[\tilde{T}_{\text {inc }}\left(\omega, a, \theta, k_{z m}\right)\right]}{\partial r}+k_{1} \frac{\partial\left[\tilde{T}_{\text {ref }}\left(\omega, a, \theta, k_{z m}\right)\right]}{\partial r} \\
\quad=k_{2} \frac{\partial\left[\tilde{T}_{\text {trans }}\left(\omega, a, \theta, k_{z m}\right)\right]}{\partial r}
\end{gathered}
$$

Combining Eqs. (A2), (A4) and (A5) one obtains a system of equations, which is then used to find the coefficients $A_{n}$ and $B_{n}$.

\section{A.2. Cavity with null fluxes along its boundary}

In this case, the incident heat field is all reflected back into the unbounded medium after reaching the inclusion surface, verifying the condition at $r=a$,

$k_{1} \frac{\partial\left[\tilde{T}_{\text {inc }}\left(\omega, a, \theta, k_{z m}\right)\right]}{\partial r}+k_{1} \frac{\partial\left[\tilde{T}_{\text {ref }}\left(\omega, a, \theta, k_{z m}\right)\right]}{\partial r}=0$

Under this condition the transmitted heat field is null. Therefore, the solution is found combining Eqs. (A2) and (A4), so as to satisfy Eq. (A7). When this is done, one obtains

$$
A_{n}=\frac{\frac{-\mathrm{i}}{4 k_{1}}(-1)^{n} \varepsilon_{n} H_{n}\left(k_{\alpha_{1}} r_{0}\right)\left[-n J_{n}\left(k_{\alpha_{1}} a\right)+\left(k_{\alpha_{1}} a\right) J_{n+1}\left(k_{\alpha_{1}} a\right)\right]}{n H_{n}\left(k_{\alpha_{1}} a\right)-\left(k_{\alpha_{1}} a\right) H_{n+1}\left(k_{\alpha_{1}} a\right)}
$$

\section{A.3. Cavity with null temperatures along its boundary}

The boundary condition at the surface of the cavity $(r=$ a) is

$\tilde{T}_{\text {inc }}\left(\omega, a, \theta, k_{z m}\right)+\tilde{T}_{\text {ref }}\left(\omega, a, \theta, k_{z m}\right)=0$

Manipulating Eqs. (A2), (A4) and (A9), the following result is obtained

$A_{n}=\frac{\frac{\mathrm{i}}{4 k_{1}}(-1)^{n} \varepsilon_{n} H_{n}\left(k_{\alpha_{1}} r_{0}\right) J_{n}\left(k_{\alpha_{1}} a\right)}{H_{n}\left(k_{\alpha_{1}} a\right)}$

\section{References}

[1] Carslaw HS, Jaeger JC. Conduction of heat in solids, 2nd ed. Oxford: Oxford University Press; 1959.

[2] Bathe KJ. Numerical methods in finite element analysis. Englewood Cliffs, NJ: Prentice-Hall; 1976.

[3] Freitas V, Abrantes V, Crausse P. Moisture migration in building walls, analysis of the interface phenomena. Bldg Environ 1996;31(2): 99-108.

[4] Brebbia CA, Telles JC, Wrobel LC. Boundary elements techniques: theory and applications in engineering. Berlin: Springer; 1984.

[5] Chang YP, Kang CS, Chen DJ. The use of fundamental green functions for solution of problems of heat conduction in anisotropic media. Int J Heat Mass Transfer 1973;16:1905-18.

[6] Shaw RP. An integral equation approach to diffusion. Int J Heat Mass Transfer 1974; 17:693-9.

[7] Wrobel LC, Brebbia CA. A formulation of the boundary element method for axisymmetric transient heat conduction. Int $\mathrm{J}$ Heat Mass Transfer 1981;24:843-50.

[8] Dargush GF, Banerjee PK. Application of the boundary element method to transient heat conduction. Int J Numer Meth Engng 1991; 31:1231-47.

[9] Wrobel LC. The boundary element method: application in thermofluids and acoustics. Sussex: Wiley; 2001.

[10] Davey K, Bounds S. Source-weighted domain integral approximation for linear transient heat conduction. Int J Numer Meth Engng 1996;39: $1775-90$.

[11] Ibañez MT, Power H. An efficient direct BEM numerical scheme for heat transfer problems using Fourier series. Int J Numer Meth Heat Fluid Flow 2000;10:687-720.

[12] Davey K, Bounds S. Source-weighted domain integral approximation for linear transient heat conduction. Int J Numer Meth Engng 1996;39: 1775-90.

[13] Bialecki RA, Jurgas P, Kuhn G. Dual reciprocity BEM without matrix inversion for transient heat conduction. Engng Anal Bound Elem 2002;26(3):227-36.

[14] Rizzo FJ, Shippy DJ. A method of solution for certain problems of transient heat conduction. AIAA J 1970;8:2004-9. 
[15] Cheng AHD, Abousleiman Y, Badmus T. A Laplace transform BEM for axysymmetric diffusion utilizing pre-tabulated Green's function. Engng Anal Bound Elem 1992;9:39-46.

[16] Zhu SP, Satravaha P, Lu X. Solving the linear diffusion equations with the dual reciprocity methods in Laplace space. Engng Anal Bound Elem 1994;13:1-10.

[17] Zhu SP, Satravaha P. An efficient computational method for nonlinear transient heat conduction problems. Appl Math Model 1996;20:513-22.

[18] Sutradhar A, Paulino GH, Gray LJ. Transient heat conduction in homogeneous and non-homogeneous materials by the Laplace transform Galerkin boundary element method. Engng Anal Bound Elem 2002;26(2):119-32.

[19] Stehfest H. Algorithm 368: numerical inversion of Laplace transform. Commun Assoc Comput Mach 1970;13(1):47-9.

[20] Tadeu A, Godinho L. 3D wave scattering by a fixed cylindrical inclusion submerged in a fluid medium. Engng Anal Bound Elem 1999;23:745-56.

[21] Tadeu A, Kausel E. Green's functions for two-and-a-half dimensional elastodynamic problems. J Engng Mech, ASCE 2000;126(10):1093-7.
[22] Tadeu A, Godinho L, Santos P. Wave motion between two fluid filled boreholes in an elastic medium. Engng Anal Bound Elem 2002;26(2): 101-17.

[23] Godinho L, António J, Tadeu A. 3D sound scattering by rigid barriers in the vicinity of tall buildings. Appl Acoust 2001;62(11): 1229-48.

[24] Bouchon M, Aki K. Time-domain transient elastodynamic analysis of 3D solids by BEM. Int J Numer Meth Engng 1977;26: 1709-28.

[25] Tadeu A, Santos P, Kausel E. Closed-form integration of singular terms for constant, linear and quadratic boundary elements. Part I. SH wave propagation. Engng Anal Bound Elem 1999;23(8): 671-81.

[26] Tadeu A, Santos P, Kausel E. Closed-form integration of singular terms for constant, linear and quadratic boundary elements. Part II. SV-P wave propagation. Engng Anal Bound Elem 1999;23(9): 757-68.

[27] Watson GN. A treatise on the theory of Bessel functions, 2nd ed. Cambridge: Cambridge University Press; 1980. 